\title{
Infección por el virus de la Hepatitis B en el embarazo
}

\author{
Luis Guillermo Toro-Rendón*
}

\begin{abstract}
*Médico hepatólogo clínico y de trasplante. Director de la Unidad Funcional de Enfermedades Digestivas de los Hospitales de San Vicente Fundación de Medellín y Rionegro. Medellín y Rionegro. Antioquia. Grupo de Investigación en Trasplante de órganos en Hospital San Vicente Fundación (INTRO). Medellín. Antioquia. Docente Universidad CES. Medellín. Antioquia. Colombia.

Correspondencia: Dr. Luis Guillermo Toro. Dirección: vereda La Convención, vía Aeropuerto - Llanogrande Km 2,3. Rionegro. Antioquia. Colombia. Teléfono: 4448717 Extensión 3111. Correo electrónico: igtr@sanvicentefundacion.com
\end{abstract}

\section{Resumen}

Introducción: la infección por el virus de la hepatitis B constituye un importante problema de salud pública a nivel mundial, pues genera una gran carga de morbilidad y mortalidad relacionada con cirrosis y hepatocarcinoma. En áreas de alta prevalencia la transmisión vertical constituye una fuente importante de infección. Objetivo: revisar la presentación de la infección por virus de la hepatitis B en el embarazo y ofrecer pautas para el manejo de esta entidad. Metodología de búsqueda: se realizó una búsqueda bibliográfica en las bases de datos Pubmed, LILACS, SciELO y el motor de búsqueda Google Scholar, de artículos publicados hasta agosto de 2017, utilizando los términos hepatitis B virus AND infection AND pregnancy. Se restringió la búsqueda a artículos de los últimos quince años, en inglés y español, incluyendo artículos de revisión, estudios clínicos controlados y metaanálisis. Se obtuvieron en total 535 manuscritos para revisión, de los cuales 52 fueron referenciados. Desarrollo del tema: los hijos de madres seropositivas para el antígeno de superficie de la hepatitis B deberían recibir inmunoglobulina y vacunación contra la hepatitis B en las primeras doce horas de nacimiento, lográndose reducir la tasa de transmisión vertical desde más del 90\% a menos del 10\%. La terapia antiviral, al ser administrada en el tercer trimestre de gestación, puede prevenir la falla inmunoprofiláctica, y debería utilizarse en madres con alto riesgo de trasmisión vertical. Datos recientes apuntan acerca de la seguridad de Tenofovir en el embarazo, siendo actualmente el medicamento de elección. Conclusiones: el tamizaje universal en la gestante para la infección por el virus de la hepatitis B es una medida costoefectiva para reducir la transmisión vertical. Los niveles elevados de antígeno de superficie del virus de la hepatitis B y la carga viral materna, así como la presencia de antígeno e, se asocian a mayor riesgo de transmisión vertical. La combinación de inmunoglobulina y vacunación para hepatitis B, administradas dentro de las doce horas posteriores al nacimiento, se asocian a reducción de la tasa de transmisión vertical. El Tenofovir es la mejor opción terapéutica como terapia antiviral iniciado en las semanas 28-32 de gestación en aquellas gestantes con alto riesgo de transmisión de la infección. MÉD.UIS. 2018;31(2):49-56.

Palabras clave: Hepatitis B. Hepatitis crónica. Embarazo. Transmisión vertical de enfermedad infecciosa. Antivirales.

\section{Infection by the Hepatitis B virus in pregnacy}

\section{Abstract}

Introduction: infection by the hepatitis B virus represents an important worldwide public health problem, since it generates a large burden of morbidity and mortality related to cirrhosis and hepatocellular carcinoma. In areas of high prevalence, vertical transmission is a major source of infection. Objective: to review the presentation of the infection by the Hepatitis B virus during pregnancy and offer guidelines to treat this entity. Search methodology: a bibliographic search was made through Pubmed, LILACS and SciELO databases, and Google Scholar search engine, of articles published until august 2017, using the terms hepatitis B virus AND infection AND pregnancy. The search was restricted to articles of the last fifteen years, in english and spanish, including review articles, controlled clinical trials and meta-analysis. A total of 535 articles were obtained for review, from which 52 were referenced. Results: children from seropositive mothers for the surface antigen of hepatitis B should receive immunoglobulin and vaccination against hepatitis $B$ in the first twelve hours after birth, reducing the vertical transmission rate from more than $90 \%$ to less than $10 \%$. Antiviral therapy on the third gestational trimester can prevent immunoprophylactic failure, and should be used on mothers at high risk of vertical transmission. Recent data point towards tenofovir's safety during pregnancy, being the treatment of choice. Conclusions: universal screening on pregnant women for the hepatitis B virus infection is a cost-effective measure to reduce vertical transmission. High hepatitis B virus surface antigen levels and mother's viral load, as well as antigen e presence, are associated with increased risk of vertical transmission. The combination of hepatitis

Artículo recibido el 11 de mayo de 2017 y aceptado para publicación el 06 de marzo de 2018 
B immunoglobulin and vaccination, given before twelve hours after birth, are associated to a reduction of the vertical transmission rate. Tenofovir is the best treatment option as antiviral therapy initiated on gestational weeks 28-32 in pregnant women at high risk of infection transmission.MÉD.UIS. 2018;31(2):49-56.

Keywords: Hepatitis B. Chronic hepatitis. Pregnancy. Infectious disease vertical transmission. Antiviral agents.

¿Cómo citar este artículo?: Toro-Rendón LG. Infección por el virus de la Hepatitis B en el embarazo. MÉD.UIS. 2018;31(2):49-56. Páginas. DOI: 10.18273/revmed.v31n2-2018006

\section{Introducción}

El Virus de la Hepatitis B (VHB) es uno de los virus más pequeños que infecta a los seres humanos. Es de tipo ADN bicatenario, envuelto, que pertenece a la familia Hepadnaviridae'. De naturaleza primariamente hepatotropo, en el hospedero se replica y se ensambla de manera exclusiva en el hepatocito y produce daño hepático por la muerte de las células infectadas, como resultado de una respuesta inmune del huésped a los antígenos virales y no por la replicación del ADN directamente. Este virus puede causar tanto hepatitis aguda como crónica ${ }^{1,2}$.

Es bien conocido que el VHB es altamente oncogénico y confiere un alto riesgo para el desarrollo de hepatocarcinoma, pues representa de modo global el 50\% de todos los casos y el $80 \%$ en áreas endémicas de $\mathrm{VHB}^{3}$.

Se estima que un tercio de la población mundial tiene evidencia serológica de infección pasada o presente por el VHB, con una prevalencia que varía según al área geográfica que se estudie, siendo más alta en África y Asia, donde supera el 5\%. Además, 350-400 millones de personas son portadoras crónicas del antígeno de superficie (HBsAg), 240 millones tiene hepatitis crónica ${ }^{4-6}$, y aunque se dispone de una vacuna segura y efectiva, cada año son diagnosticados 50 millones de casos nuevos a nivel mundial ${ }^{4}$, la mayoría adquiridos por transmisión vertical, especialmente en regiones de alta prevalencia?.

Todas las mujeres en gestación deberían ser tamizadas en el primer trimestre para $\mathrm{HBsAg}^{8}$, pues esta estrategia ha demostrado ser costo-efectiva para la prevención de la transmisión vertical y es la intervención que mayor impacto ha tenido en disminuir el riesgo de la infección neonatal ${ }^{9}$, teniendo en cuenta que en Estados Unidos se ha estimado una prevalencia de la infección en un 0,7-0,9 de las mujeres en gestación, con más de 25000 niños en riesgo de infección nacidos anualmente ${ }^{10}$.
La infección crónica, definida como la persistencia del HBsAg por más de 6 meses, es inversamente proporcional a la edad al momento de la exposición al virus, presentando hepatitis crónica el 90\% de los lactantes nacidos de madres antígeno $\mathrm{E}$ positivas (HBeAg), 50\% de los niños pequeños expuestos, pero solo $5 \%$ de los adultos 5 .

El embarazo no afecta a la enfermedad hepática relacionada con el VHB, aunque se han reportado que los casos de exacerbación de la hepatitis, los cambios relacionados con el medio hormonal y las citoquinas pueden llevar a fluctuaciones de la carga viral y de las enzimas hepáticas. Se ha descrito que después del parto puede presentarse un aumento de la actividad de la enfermedad hepática en ausencia de tratamiento antiviral, lo cual podría estar relacionado a una reactivación del sistema inmune, por lo que se recomienda un seguimiento estrecho de estas pacientes después de la gestación $n^{11,12}$.

La infección por VHB es una entidad altamente prevalente en el mundo, lo cual se traduce en una alta posibilidad de transmisión perinatal, pues, sin un adecuado manejo de esta patología, hasta el 90\% de los neonatos pueden desarrollar una infección crónica. Teniendo esto en cuenta, el propósito de este artículo es revisar la presentación de la infección por virus de la hepatitis $B$ en el embarazo y ofrecer pautas actualizadas para su manejo.

\section{Metodología de búsqueda}

Se realizó una búsqueda bibliográfica utilizando los términos hepatitis $B$ virus AND infection AND pregnancy en las bases de datos Pubmed, LILACS, SciELO y el motor de búsqueda Google Scholar, de artículos publicados hasta el 28 de agosto de 2017. La búsqueda se restringió a un periodo de tiempo de los últimos quince años, aunque se revisaron algunos artículos clásicos de años anteriores. También se limitó a estudios en seres humanos, para los idiomas 
Mayo - agosto

inglés y español, artículos de revisión, estudios clínicos controlados y metaanálisis. Se obtuvieron en total 535 artículos, cuyo resumen fue revisado, de los cuales 52 fueron referenciados por su importancia para la revisión actual y su alta calidad metodológica.

\section{Desarrollo del tema}

\section{Rutas de transmisión del VHB}

El VHB se transmite predominantemente por contacto con sangre o ciertos fluidos corporales contaminados (menstrual, vaginal, seminal), del mismo modo que el virus de la inmunodeficiencia humana (VIH), teniendo el VHB una eficiencia en su transmisión 50 a 100 veces superior al VIH. Las concentraciones virales más altas están presentes en la sangre y los fluidos serosos, mientras que las concentraciones más bajas se encuentran en la saliva y el semen.

La transmisión sexual es más frecuente en los hombres no vacunados que tienen relaciones sexuales con hombres, personas heterosexuales con múltiples parejas sexuales, o aquellos que tienen contacto con trabajadoras sexuales. La transmisión del virus también puede ocurrir por transfusiones sanguíneas, inoculación de pequeñas cantidades de sangre o fluido durante procedimientos quirúrgicos o dentales, uso de navajas y de otros objetos similares contaminados con sangre, uso de jeringas y agujas inadecuadamente esterilizadas en usuarios de drogas intravenosas o durante la realización de un tatuajes, piercing y en procedimientos de acupuntura, cuando los instrumentos están contaminados ${ }^{3}$.

En los países donde el VHB es endémico, la mayoría de las infecciones son adquiridas a través de la exposición perinatal, siendo reportadas tasas de transmisión madre a hijo (TMH) hasta en un 70-90\% cuando la madre es HBsAg y HBeAg positiva y hay ausencia de profilaxis post-exposición en el lactante ${ }^{13}$.

La transmisión horizontal también es importante, incluyendo la intrafamiliar y de especial valor, niño a niño, encontrando que al menos el $50 \%$ de las infecciones en los infantes no pueden explicarse por $\mathrm{TMH}$. En áreas endémicas, antes de la introducción de la vacunación neonatal, el pico de incidencia de este tipo de transmisión se ubicaba entre los 7-14 años ${ }^{3}$.

\section{Hepatitis b aguda durante el embarazo}

El curso de la infección aguda por VHB durante el embarazo es similar al de la población general. El período de incubación oscila entre seis semanas a seis meses (promedio de 75 días) y sólo el $50 \%$ de las personas infectadas presentan síntomas como ictericia, náuseas, vómito, dolor abdominal y fatiga. El riesgo de falla hepática después de una infección aguda es de aproximadamente el $1 \%$.

Al igual que la población general, $95 \%$ de las gestantes coninfección aguda presentan resolución espontánea de esta, y el tratamiento es básicamente de soporte. Sin embargo, cuando ocurre en las primeras semanas de gestación, podría causar aborto espontáneo. Por otra parte, cuando el inicio de la infección aguda es al final del embarazo, la transmisión vertical es mucho más frecuente y se puede asociar con la presencia de bajo peso al nacer ${ }^{14}$. La tasa de TMH en el contexto de infección aguda por VHB es del 10\% si la infección materna ocurre en etapas tempranas del embarazo, pero aumenta hasta el $60 \%$ si la infección materna ocurre en el momento del parto o cerca de éste ${ }^{15}$.

\section{Transmisión de la madre al hijo}

El riesgo de transmisión vertical es aumentado por factores como:

- Carga viral de VHB en la madre: la alta viremia materna se correlaciona con un mayor riesgo de transmisión de la infección durante el embarazo ${ }^{16-18}$, tal como se evidencia en un estudio retrospectivo publicado por $\mathrm{H}$. Zoul et al en 2012, que evaluó la posibilidad de falla de la inmunoprofilaxis (uso de inmunoglobulina y vacunación en el recién nacido) y su relación con la carga viral materna en 869 niños nacidos de madres HBsAg positivas, encontrando una falla en la inmunoprofilaxis en el 3,1\% de los niños, lo cual se asoció de manera significativa con los valores de la carga viral materna. La tasa de TMH según la carga viral materna más frecuente fue $8 \log _{10}$ copias $/ \mathrm{ml}(7,6 \%)$, mientras que la menos frecuente fue de $<6 \log _{10}$ copias $/ \mathrm{ml}(0 \%)^{3}$. El análisis de regresión logística multivariada identificó los niveles de carga viral materna (OR=1,85, IC 95\%: 1,07-3,30) y la presencia de carga viral detectable en la sangre del cordón (OR=39,67, IC 95\%: 14,22-110,64), como factores 
de riesgo independientes para falla en la inmunoprofilaxis ${ }^{19}$.

De igual forma, un estudio prospectivo de 303 gestantes con infección por VHB publicado por Wen HN et al en 2013, también demostró que la carga viral materna se asocia de manera significativa con el riesgo de infección (por cada incremento de $\log _{10}$ copias/ml: OR: 3,49, IC 95\%: 1,63-7,48, p=0,001). En ambos estudios, todas las fallas a la inmunoprofilaxis se dieron cuando la carga viral materna fue $>6 \log _{10}$ copias $/ \mathrm{ml}^{17,19}$.

- HBeAg materno: el HBeAg es considerado un marcador de replicación e infectividad, y su presencia se asocia usualmente con cargas virales elevadas. En ausencia de inmunoprofilaxis, la transmisión perinatal ocurre en $>90 \%$ si la madre es HBeAg positiva y en el $15 \%$ si es HBeAg negativa; mientras que, después de una adecuada inmunoprofilaxis la tasa de transmisión perinatal cae al $2 \%$ de los infantes, y la gran mayoría de estos casos se da cuando las cargas virales son superiores a $200000 \mathrm{UI} / \mathrm{ml}$ $\left(10^{6} \text { copias } / \mathrm{ml}\right)^{15,20}$.

- Profilaxis post-exposición: la inmunoprofilaxis activa-pasiva con la vacuna contra la hepatitis B y la inmunoglobulina contra la hepatitis B (HBIG) tiene un efecto dramático sobre las tasas de $\mathrm{TMH}$, que varían marcadamente según el estado del $\mathrm{HBeAg}$ de la madre ${ }^{13}$. De esta forma, la combinación de HBIG y vacunación, administradas dentro de las doce horas posteriores al nacimiento, ha reducido efectivamente la tasa de transmisión perinatal de más del $90 \%$ sin inmunoprofilaxis a menos de $10 \%$ cuando esta se administra ${ }^{15}$.

\section{Tratamiento}

La FDA ha dividido los medicamentos en cinco categorías para su uso durante el embarazo (Ver Tabla 1). Ninguno de ellos es categoría A para el tratamiento de VHB. Sin embargo, en la categoría B y $C$ hay algunas alternativas disponibles.

Actualmente hay dos opciones principales de tratamiento para la infección crónica por VHB: el Interferón pegilado a (PegINFa), los análogos nucleósidos: Lamivudina (3TC), Telbivudina (TBV), Entecavir (ETV) análogos nucleótidos: Adefovir
(ADV), Tenofovir (TDF) (Ver Tabla 2). Los análogos nucleósidos/nucleótidos, pueden ser clasificados como antivirales con una baja barrera genética contra el VHB (3TC, ADV, TBV), con tasas de resistencia tan altas como hasta del $70 \%$ a 5 años, como en el caso de la 3TC; y aquellos con una alta barrera genética (ETV, TDF), con tasas de resistencia a 5 años sumamente bajas (2\% para el ETV, por ejemplo). La principal ventaja del tratamiento con análogos nucleósidos/ nucleótidos es su eficacia antiviral, siendo previsible a largo plazo y llevando a niveles indetectables de ADN de VHB en la gran mayoría de los casos; así como su mayor seguridad².

Tabla 1. Clasificación de los medicamentos en categorías de riesgo en el embarazo según la FDA.

\begin{tabular}{|c|c|}
\hline \multicolumn{2}{|c|}{$\begin{array}{l}\text { Clasificación de los medicamentos según la FDA en } \\
\text { categorías de riesgo en el embarazo }\end{array}$} \\
\hline Categoría & Descripción de la FDA \\
\hline A & $\begin{array}{l}\text { Estudios adecuados y bien controlados no han } \\
\text { demostrado un riesgo para el feto en el primer } \\
\text { trimestre del embarazo y no hay evidencia de } \\
\text { riesgo en los últimos trimestres. }\end{array}$ \\
\hline B & $\begin{array}{l}\text { Estudios en animales han mostrado que no } \\
\text { existe evidencia de daño al feto. Sin embargo, } \\
\text { no hay estudios adecuados y bien controlados en } \\
\text { mujeres embarazadas }\end{array}$ \\
\hline C & $\begin{array}{l}\text { Estudios de reproducción animal han demostrado } \\
\text { algún efecto adverso en el feto, no hay estudios } \\
\text { adecuados y bien controlados en seres humanos, } \\
\text { pero los beneficios potenciales podrían justificar } \\
\text { el uso del medicamento en mujeres embarazadas } \\
\text { a pesar de los riesgos potenciales. }\end{array}$ \\
\hline D & $\begin{array}{l}\text { Los estudios controlados y observacionales } \\
\text { realizados en mujeres embarazadas han } \\
\text { demostrado algún riesgo para el feto. Sin } \\
\text { embargo, el beneficio de su uso en mujeres } \\
\text { embarazadas puede aceptarse a pesar el riesgo. } \\
\text { Su uso en la embarazada está justificado cuando } \\
\text { exista riesgo vital, o no se puedan prescribir } \\
\text { fármacos más seguros o son ineficaces. }\end{array}$ \\
\hline$x$ & $\begin{array}{l}\text { Los estudios controlados y observacionales } \\
\text { realizados en animales o en seres humanos } \\
\text { han demostrado una clara evidencia de } \\
\text { anormalidades o riesgo para el feto. El riesgo de } \\
\text { uso del medicamento en la mujer embarazada } \\
\text { sobrepasa claramente cualquier beneficio. El } \\
\text { medicamento está contraindicado en la mujer } \\
\text { que esta o pueda quedar embarazada. }\end{array}$ \\
\hline
\end{tabular}
2012; 10:452-9 
Tabla 2. Medicamentos aprobados para el tratamiento de la infección por VHB en adultos.

\begin{tabular}{|c|c|c|l|l|}
\hline \multicolumn{7}{|c|}{ Medicamentos aprobados para el tratamiento de la infección por VHB en adultos } \\
\hline Medicamento & $\begin{array}{c}\text { Dosis en } \\
\text { adultos }\end{array}$ & $\begin{array}{c}\text { Categoría en } \\
\text { el embarazo }\end{array}$ & \multicolumn{1}{|c|}{ Potenciales efectos adversos } & \multicolumn{1}{c|}{ Uso en el embarazo } \\
\hline $\begin{array}{c}\text { Interferón pegilado a2a } \\
\text { (Peg-INF a2a) }\end{array}$ & $\begin{array}{c}180 \mathrm{mgr} / \\
\text { semana }\end{array}$ & C & $\begin{array}{l}\text { Síntomas Flu-Like, fatiga, citopenias, } \\
\text { enfermedades autoinmunes }\end{array}$ & No recomendado \\
\hline Lamivudina (3TC) & $100 \mathrm{mg} /$ día & C & Pancreatitis, acidosis láctica & $\begin{array}{l}\text { Amplios datos de seguridad en humanos. } \\
\text { Altas tasas de resistencia viral }\end{array}$ \\
\hline Telbivudina (TBV) & $600 \mathrm{mg} / \mathrm{día}$ & B & $\begin{array}{l}\text { Miopatía, elevación de la } \\
\text { creatinquinasa, neuropatía periférica }\end{array}$ & $\begin{array}{l}\text { Datos positivos sobre la seguridad en } \\
\text { humanos. Menos datos que con 3TC o TDF }\end{array}$ \\
\hline Entecavir (ETV) & $0.5-1 \mathrm{mg} /$ \\
día* & C & Acidosis láctica & No recomendado \\
\hline Adefovir (ADV) & $10 \mathrm{mg} / \mathrm{día}$ & C & $\begin{array}{l}\text { Falla renal aguda, síndrome } \\
\text { de Fanconi, diabetes insípida } \\
\text { nefrogénica, acidosis láctica }\end{array}$ & No recomendado \\
\hline Tenofovir (TDF) & $300 \mathrm{mg} / \mathrm{día}$ & B & $\begin{array}{l}\text { Síndrome de Fanconi, osteomalacia, } \\
\text { acidosis láctica }\end{array}$ & Amplios datos de seguridad en humanos \\
\hline
\end{tabular}

Fuente: tomado y modificado de: AASLD Guidelines for Treatment of Chronic Hepatitis B ${ }^{4}$.

El PegIFNa está contraindicado durante el embarazo. No hay estudios adecuados y bien controlados con ADV y ETV en mujeres embarazadas, por lo que no se recomienda su uso en este grupo de pacientes. Por otra parte, se han realizado estudios de reproducción en animales y en seres humanos con 3TC, TDF y TBV, que no han evidenciado daño al feto debido a estos fármacos ${ }^{21-23}$. Entre los tres últimos agentes, el TDF debe ser preferido, ya que tiene un mejor perfil de resistencia y datos de seguridad más amplios en mujeres embarazadas con infección por $\mathrm{VHB}^{2}$.

En una cohorte de madres HBeAg positivas que tenían niveles de ADN del VHB superiores a $200000 \mathrm{UI} / \mathrm{ml}$ durante el tercer trimestre, la tasa de TMH fue más baja entre aquellas que recibieron tratamiento con TDF 0\% versus las que recibieron la terapia estándar de cuidado (vacunación e HBIG) sin antiviral, que fue del $7 \%$. Los perfiles de seguridad en la madre y el niño fueron similares tanto en el grupo de TDF como en el grupo control, incluyendo las tasas de anormalidades congénitas (2\% [2/95 lactantes] y $1 \%[1 / 88]$, respectivamente, $p=1,00)$. En la semana 28 posparto, las medidas antropométricas de los lactantes con exposición a TDF fueron similares a las del grupo control: peso: 9,12 $\pm 1,33 \mathrm{~kg}$ versus 9,25 \pm $1,46 \mathrm{~kg}(p=0,51)$, talla: $69,41 \pm 3,57 \mathrm{~cm}$ versus $69,21 \pm$ $3,84 \mathrm{~cm}(p=0,72)$ y circunferencia craneana: 44,22 \pm $2,37 \mathrm{~cm}$ vs $44,0 \pm 2,16 \mathrm{~cm}(p=0,52)^{22}$.

Los principales objetivos del tratamiento de la infección crónica por VHB en el embarazo son mantener la función hepática estable en la madre y prevenir la infección neonatal. Por lo tanto, los niveles de aminotransferasas deberán ser evaluados regularmente durante la gestación ${ }^{24}$.

Hay dos indicaciones para el tratamiento de la infección crónica por VHB en las mujeres gestantes (Ver Figura 1)

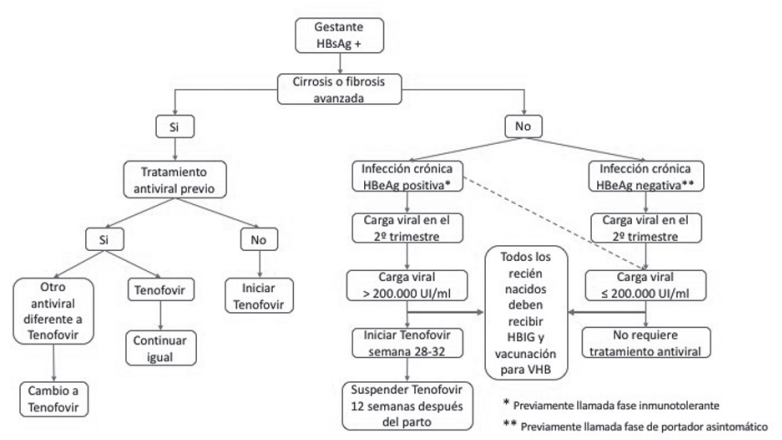

Figura 1: enfoque de la paciente gestante con $\mathrm{HBsAg}$ positivo. HBsAg: antígeno de superficie de hepatitis $B$; $\mathrm{HBeAg}$ : antígeno $\mathrm{E}$ de Hepatitis B; HBIG: inmunoglobulina contra la hepatitis B; VHB: Virus de la Hepatitis B.

Fuente: modificado de: J Clin TransI Hepatol ${ }^{49}$.

1. Pacientes con infección crónica por VHB con hepatitis activa: las indicaciones de tratamiento son las mismas que en la población general: los pacientes sin cirrosis deben ser considerados para tratamiento cuando la carga viral del VHB es superior $2000 \mathrm{UI} / \mathrm{ml}$, los niveles séricos de Alanino Aminotransferasa (ALT) están por 
encima del límite del valor normal (ULN) $=40$ $\mathrm{UI} / \mathrm{ml}$ y hay evidencia de necroinflamación y/o fibrosis moderadas a la biopsia hepática. Por otra parte, los pacientes con carga viral $>20$ ooo UI/ml y ALT dos veces ULN pueden iniciar el tratamiento antiviral incluso sin una biopsia hepática. Finalmente, aquellos pacientes con fibrosis avanzada o cirrosis que ya reciben tratamiento con TDF, deberían continuar con este, pero si vienen en manejo con ETV, se deberá cambiar a TDF².

2. Prevención de la transmisión del VHB de la madre al feto: se basa en la combinación de HBIG y vacunación administradas dentro de las primeras 12 horas después del nacimiento 25,26 , manejo que deberá recibir todo niño nacido de madre $\mathrm{HBsAg}$ positivo, independientemente de que ella reciba o no tratamiento antiviral. La vacunación del recién nacido para VHB deberá continuarse hasta finalizar el esquema.

Estudios previos han demostrado que el uso de la HBIG aplicada a la madre antes del parto podría llevar a una disminución $>2 \log _{10}$ en la carga viral materna para VHB, pero con una modesta reducción en las tasas de transmisión al feto ${ }^{27}$. Sin embargo, en un estudio controlado se demostró que el uso de HBIG antes del parto no era efectivo para prevención, hallazgos que se soportan en un metaanálisis reciente $^{28,29}$. Por tanto, esta estrategia no se recomienda actualmente.

Hay un grupo de pacientes de alto riesgo de falla (tan alto como del 30\%) cuando se usa solo inmunoprofilaxis, por lo cual se benefician del uso adicional de medicación antiviral ${ }^{30,31}$. Cuando se toma la decisión de ofrecer profilaxis, el medicamento debe iniciarse entre las 28 y 32 semanas de gestación, momento en el cual la organogénesis ya está completa y se cuenta con suficiente tiempo para lograr que disminuyan los niveles de ADN del $\mathrm{VHB}^{24}$. Esta intervención ha demostrado ser costoefectiva32, y 3 TC, TBV y TDF tienen un aceptable perfil de seguridad en el feto ${ }^{33}$. La decisión de brindar tratamiento se basa en la presencia de HBsAg positivo con una carga viral $>200000 \mathrm{UI} / \mathrm{ml}\left(>10^{6}\right.$ copias) $\mathrm{ml})^{15}$. La profilaxis antiviral se deberá continuar hasta 12 semanas después del parto, debido a la posibilidad de Flare de $\mathrm{VHB}^{2,24,34}$, el cual es mucho más frecuente en las pacientes $\mathrm{HBeAg}$ positivas, con un riesgo que es 2,56 veces mayor cuando se compara con las HBeAg negativas ${ }^{35}$. Sin embargo, en las pacientes con infección crónica por $\mathrm{VHB}$ HBeAg negativo (antes llamado estado de portador asintomático) ${ }^{2}$ con cargas virales $\leq 200000 \mathrm{UI} / \mathrm{ml}$, no está indicada la profilaxis antiviral24.

Los niveles de HBsAg materno pueden ser también predictores de $\mathrm{TMH}$, como lo es la carga viral. Hay estudios que apoyan el inicio de profilaxis antiviral con niveles de $\mathrm{HBsAg}>4-4,5 \log _{10} \mathrm{UI} / \mathrm{ml}$. El riesgo de TMH aumenta de manera significativa conforme se incrementan los niveles de $\mathrm{HBsAg}$, siendo este del 0,66 para $\log _{10} \mathrm{UI} / \mathrm{ml}$ (IC 95\%: -0,4-1,6, $\left.p=0,22\right)$, mientras que para $5 \log _{10} \mathrm{UI} / \mathrm{ml}$ es de $26.4 \%$ (IC 95\%: $12,6-40,2, p<0,001)^{2,36}$.

La 3 TC ha sido ampliamente utilizada en la prevención de la transmisión perinatal del VHB. El primer estudio sobre el uso de este medicamento en el embarazo se publicó en 2003, en el cual ocho mujeres con niveles elevados de ADN del VHB fueron tratadas con lamivudina $150 \mathrm{mg} /$ día a partir de la semana 34 de gestación; 25 niños nacidos de mujeres no tratadas sirvieron como controles. Todos los niños recibieron inmunización activa y pasiva al nacer. A los 12 meses de edad, 1 de cada 8 niños (12,5\%) en el grupo de tratamiento se mantuvo HBsAg positivo con niveles positivos de ADN del VHB en comparación con 7 de 25 niños (28\%) en el grupo de control, no se observaron eventos adversos ${ }^{37}$. Sin embargo, se reconoce ahora que el uso de este fármaco limita las opciones futuras de tratamiento materno, ya que incluso el uso a corto plazo promueve variantes virales resistentes a la 3TC en al menos el $20 \%$ de las pacientes que la reciben ${ }^{38}$.

En cuanto a la TBV, en un estudio abierto de 135 mujeres con niveles de ADN del VHB $>7 \log _{10}$ copias/ml que recibieron $600 \mathrm{mg} /$ día de TBV de la semana 20 a la 32 de gestación se compararon con 94 mujeres no tratadas. Todos los recién nacidos recibieron inmunización pasiva y activa estándar al nacer. A los 7 meses después del parto, la infección por VHB fue significativamente menor en los niños nacidos de las madres tratadas con TBV comparadas con los controles ( $0 \%$ versus $8 \%$ ). No se informaron anomalías congénitas 39 . Una preocupación con respecto a la resistencia a la telbivudina en general es que sólo se necesita una sustitución en el sitio activo de la polimerasa (mutación en la región YMDD) para inducir resistencia, lo cual hace que tenga una barrera genética inferior a la del TDF o ETV, de igual forma, un problema adicional es que esta mutación también 
reduce la susceptibilidad a otros análogos como la 3TC o el ETV, debido a resistencias cruzadas, lo que puede limitar futuras opciones de tratamiento ${ }^{40,41}$.

Por lo tanto, en la actualidad, el TDF representa la opción más razonable para el tratamiento de la infección por VHB en mujeres embarazadas, por el beneficio materno y fetal, ya que es el análogo nucleósido más potente, con la menor tasa de resistencia y con datos de seguridad disponibles para su uso durante el embarazo 15,22,42,43.

La lactancia materna no está contraindicada en mujeres con HBsAg positivo no tratadas ${ }^{44}$, como es posible evidenciar en un metanálisis de diez ensayos clínicos controlados, en los que se incluyeron 1624 lactantes divididos en dos grupos: 751 en el grupo que amamantaba y 873 en el grupo que no amamantaba, encontrándose que el OR de la TMH de VHB en el grupo de lactancia materna versus el grupo no lactante era de 0,86 (IC: 95\%: 0,51-1,45), este estudio concluye que la lactancia materna después de una inmunoprofilaxis adecuada no contribuyó a la $\mathrm{TMH}$ del $\mathrm{VHB}^{45}$. Tampoco puede considerarse una contraindicación la lactancia en las madres positivas para $\mathrm{HBsAg}$ que reciben un agente antiviral como el TDF ${ }^{2,46}$. De hecho, aunque el riesgo de exposición in útero a los fármacos es probablemente mayor que el de los lactantes a través de la leche materna, estos fármacos se recomiendan para su uso durante el embarazo ${ }^{47,48}$. Sin embargo, debería evitarse en presencia de pezones agrietados o sangrantes, ya que esto podría causar la mezcla de exudados serosos con la leche materna y conducir potencialmente a la transmisión del VHB ${ }^{49}$.

Ensayos clínicos controlados no han demostrado el beneficio del parto por cesárea para prevenir la $\mathrm{TMH}^{50,51}$. Por lo cual, actualmente no se recomienda.

Es fundamental confirmar la respuesta clínica a la vacunación en recién nacidos de madres HBsAg positivo, a partir del desarrollo de títulos adecuados de anticuerpos contra el antígeno de superficie, aquellos no respondedores y los no vacunados, requerirán seguimiento por personal médico capacitado (incluyen Pediatría, Gastroenterología pediátrica, Infectología pediátrica o Hepatología) ${ }^{52}$.

\section{Conclusiones}

Los niveles elevados del antígeno de superficie y de la carga viral materna, así como la presencia de
Antígeno $\mathrm{E}$, se asocian a mayor riesgo de transmisión vertical, siendo el tamizaje universal en la gestante la medida más eficiente para disminuir la posibilidad de transmisión madre e hijo del virus de la Hepatitis B. De igual forma, la combinación de inmunoglobulina y vacunación para hepatitis $B$, administradas dentro de las 12 horas posteriores al nacimiento, se asocian a una reducción significativa en las tasa de transmisión vertical. Si hay indicación de utilizar un antiviral de alta barrera genética, el Tenofovir es la mejor opción terapéutica al ser iniciado en las semanas 28-32 de gestación en todas aquellas gestantes que tengan alto riesgo de transmisión de la infección. Por otra parte, se ha demostrado que el parto por cesárea no reduce la incidencia de transmisión perinatal del virus y no hay contraindicación para lactancia, incluso ante la toma de antivirales por la madre, siendo la única excepción la pesencia de pezones agrietados o sangrantes que eventualmente pueden favorecer la transmisión del virus.

\section{Referencias bibliográficas}

1. Rac Martha W.F SJS. Prevention and Management of Viral Hepatitis in Pregnancy. Obs Gynecol Clin N Am. Elsevier Inc; 2014;41:573-92.

2. Lampertico P, Agarwal K, Berg T, Buti M, Janssen HLA, Papatheodoridis G, et al. EASL 2017 Clinical Practice Guidelines on the management of hepatitis B virus infection. J Hepatol. 2017;67(2):370-398

3. World Health Organizaton. Guidelines for the Prevention, Care and Treatment of Persons with Chronic Hepatitis B Infection. Guidel Prev Care Treat Pers with Chronic Hepat B Infect [Internet]. 2015 [citado el ]. Disponible en: http://apps.who.int/ iris/bitstream/handle/10665/154590/9789241549059_eng.pdf;jses sionid =769197BF35F15E266EABE3B375BFAE49? sequence $=1$

4. Terrault NA, Bzowej NH, Chang K-M, Hwang JP, Jonas MM, Murad MH. AASLD guidelines for treatment of chronic hepatitis B. Hepatology. 2015;63(1):261-83

5. Association E. EASL clinical practice guidelines: Management of chronic hepatitis B virus infection. J Hepatol. European Association for the Study of the Liver; 2012;57(1):167-85.

6. Lavanchy D. Hepatitis B virus epidemiology, disease burden, treatment, and current and emerging prevention and control measures. J Viral Hepat. 2004;11:97-107.

7. Tran TT. Hepatitis B in Pregnancy. Clin Infect Dis. 2016;62(4):31417.

8. Kubo A, Shlager L, Marks AR, Lakritz D, Colette Beaumont, Gabellini K, et al. Prevention of Vertical Transmission of Hepatitis B: An Observation Study. Ann Intern Med. 2014;160(12):828-35.

9. Chamroonkul N, Piratvisuth T. Hepatitis B During Pregnancy in Endemic Areas: Screening, Treatment, and Prevention of Motherto-Child Transmission. Pediatr Drugs. 2017;19(3):173-81.

10. Dionne-odom J, Tita A, Silverman NS. Hepatitis B in pregnancy screening, treatment, and prevention of vertical transmission. Am J Obstet Gynecol. 2016;214(1):6-14.

11. Kar P, Mishra S. Management of hepatitis B of during pregnacy, Expert Opin Pharmacother. 2016;17(3):301-10.

12. Ter MJ, Leemans WF, De Man RA, Janssen HLA. Exacerbation of chronic hepatitis B infection after delivery. J Viral Hepat. 2008;15(1):37-41.

13. Patton H, Tran TT. Management of hepatitis B during pregnancy. Nat Rev Gastroenterol Hepatol. 2014;11(7):402-9.

14. Pol S, Corouge M, Fontaine H. Hepatitis B virus infection and pregnancy. Clin Res Hepatol Gastroenterol. 2011;35(10):618-22.

15. Castillo E, Murphy K. Hepatitis B and Pregnancy. J Obs Gynecol. 
2017;39(3):181-90.

16. Liu C-P, Zeng Y-L, Zhou M, Chen L-L, Hu R, Wang L, et al. Factors associated with mother-to-child transmission of hepatitis B virus despite immunoprophylaxis. Intern Med. 2015;54(7):711-6.

17. Wen WH, Chang MH, Zhao LL, Ni YH, Hsu HY, Wu JF, et al. Motherto-infant transmission of hepatitis B virus infection: Significance of maternal viral load and strategies for intervention. J Hepatol. 2013;59(1):24-30.

18. Lu L-L, Chen B-X, Wang J, Wang D, Ji Y, Yi H-G, et al. Maternal transmission risk and antibody levels against hepatitis $\mathrm{B}$ virus e antigen in pregnant women. Int J Infect Dis. 2014;28:41-4.

19. Zou H, Chen Y, Duan Z, Zhang H, Pan C. Virologic factors associated with failure to passive-active immunoprophylaxis in infants born to HBsAg-positive mothers. J Viral Hepat. 2012;19(2):18-25.

20. Ott J, Stevens G, Wiersma S. The risk of perinatal hepatitis B virus transmission: hepatitis B e antigen (HBeAg) prevalence estimates for all world regions. BMC Infect Dis. 2012;12(12):131

21. Wu Q, Huang H, Sun X, Pan M, He Y, Tan S, et al. Telbivudine Prevents Vertical Transmission of Hepatitis B Virus From Women With High Viral Loads: A Prospective Long-Term Study. Clin Gastroenterol Hepatol. 2015;13(6):1170-6.

22. Han G, Wang Y, Zhang H, Zou H, Zhu B, Zhao W. Tenofovir to Prevent Hepatitis B Transmission in Mothers with High Viral Load. N Engl J Med. 2016;374(24):2324-34.

23. Deng M, Zhou X, Gao S, Yang S-G, Wang B, Chen H-Z, et al. The effects of telbivudine in late pregnancy to prevent intrauterine transmission of the hepatitis B virus: a systematic review and meta-analysis. Virol J. Virology Journal. 2012;9(185):1-13.

24. Shao Z, Al Tib M, Wakim-Fleming J. Update on viral hepatitis in pregnancy. Cleve Clin J Med. 2017;84(3):202-6.

25. Lee C, Gong Y, Brok J, Boxall EH, Gluud C. Hepatitis B immunisation for newborn infants of hepatitis B surface antigenpositive mothers. Cochrane database Syst Rev. 2006;(2):1-107.

26. Beasley RP, Hwang LY, Lee GC, Lan CC, Roan CH, Huang FY, et al. Prevention of perinatally transmitted hepatitis B virus infections with hepatitis B immune globulin and hepatitis B vaccine. Lancet. 1983;12(8359):1099-102.

27. Li XM, Shi MF, Yang YB, Shi ZJ, Hou HY, Shen HM, et al. Effect of hepatitis B immunoglobulin on interruption of HBV intrauterine infection. World J Gastroenterol. 2004;10(21):3215-7.

28. Yuan J, Lin J, Xu A, Li H, Hu B, Chen J, et al. Antepartum immunoprophylaxis of three doses of hepatitis B immunoglobulin is not effective: A single-centre randomized study. J Viral Hepat. 2006;13(9):597-604.

29. Ac E, Gu E, Ua E, Xia Y, Liu J. Hepatitis B immunoglobulin during pregnancy for prevention of mother-to-child transmission of hepatitis B virus. Cochrane Database Syst Rev. 2017;(2):1-103.

30. Pan CQ, Duan ZP, Bhamidimarri KR, Zou HBIN, Liang XF, Li JIE. An Algorithm for Risk Assessment and Intervention of Mother to Child. Clin Gastroenterol Hepatol. 2012;10(5):452-9.

31. Brown RS, McMahon BJ, Lok ASF, Wong JB, Ahmed AT, Mouchli MA, et al. Antiviral therapy in chronic hepatitis B viral infection during pregnancy: A systematic review and meta-analysis Hepatology. 2016;63(1):319-33.

32. Wang W, Wang J, Dang S, Zhuang G. Cost-effectiveness of antiviral therapy during late pregnancy to prevent perinatal transmission of hepatitis B virus. PeerJ. 2016;24(4):1-19.

33. Sarkar M, Terrault NA. Ending vertical transmission of hepatitis B The third trimester intervention. Hepatology. 2014;60(2):448-51.

34. Chang CY, Aziz N, Poongkunran M, Javaid A, Trinh HN, Lau D, et al. Serum Alanine Aminotransferase and Hepatitis B DNA Flares in Pregnant and Postpartum Women with Chronic Hepatitis B. Am J Gastroenterol. 2016;111(10):1-6.

35. Giles M, Visvanathan K, Lewin S, Bowden S, Locarnini S, Spelman T, et al. Clinical and virological predictors of hepatic flares in pregnant women with chronic hepatitis B. Gut.
2014;64(11):1810-5.

36. Wen WH, Huang CW, Chie WC, Yeung CY, Zhao LL, Lin WT, et al. Quantitative maternal hepatitis B surface antigen predicts maternally transmitted hepatitis B virus infection. Hepatology. 2016;64(5):1451-61.

37. Zonneveld M, Nunen A, Niesters HGM, Man R., Schalm SW, Janssen HL. Lamivudine treatment during pregnancy to prevent perinatal transmission of hepatitis B virus infection. J Viral Hepat. 2003;10(4):294-7.

38. Ayres A, Yuen L, Jackson KM, Manoharan S, Glass A, Maley M, et al. Short duration of lamivudine for the prevention of hepatitis $B$ virus transmission in pregnancy: Lack of potency and selection of resistance mutations. J Viral Hepat. 2014;21(11):809-17.

39. Han GR, Cao MK, Zhao W, Jiang HX, Wang CM, Bai SF, et al. A prospective and open-label study for the efficacy and safety of telbivudine in pregnancy for the prevention of perinatal transmission of hepatitis B virus infection. J Hepatol. European Association for the Study of the Liver. 2011;55(6):1215-21.

40. Jaffe A, Brown RS. A Review of Antiviral Use for the Treatment of Chronic Hepatitis B Virus Infection in Pregnant Women. Gastroenterol Hepatol (N Y). 2017;13(3):154-63.

41. Seifer M, Patty A, Serra I, Li B, Standring DN. Telbivudine, a nucleoside analog inhibitor of HBV polymerase, has a different in vitro cross-resistance profile than the nucleotide analog inhibitors adefovir and tenofovir. Antiviral Res. 2009;81(2):147-55.

42. Chen J, Liao Z, Huang F, Su RK, Wang W, Cheng X, et al. Efficacy and safety of tenofovir disoproxil fumarate in preventing vertical transmission of hepatitis B in pregnancies with high viral load. Sci Rep. Springer US; 2017;7(1):4132.

43. Jourdain G, Ngo-Giang-Huong N, Cressey TR, Hua L, Harrison L, Tierney C, et al. Prevention of mother-to-child transmission of hepatitis B virus: a phase III, placebo-controlled, double-blind, randomized clinical trial to assess the efficacy and safety of a short course of tenofovir disoproxil fumarate in women with hepatitis B virus . BMC Infect Dis. BMC Infectious Diseases; 2016;16:393.

44. Hill J, Sheffield J, Kim J E. Risk of hepatitis B transmission in breast-fed infants of chronic hepatitis B carriers. Gynecol Obs. 2002;99(6):1049-52.

45. Shi Z. Breastfeeding of Newborns by Mothers Carrying Hepatitis B Virus. Arch Pediatr Adolesc Med. 2011;165(9):837

46. The American Academy of Pediatrics. Breastfeeding and the Use of Human Milk. Pediatrics. 2012;129(3):827-41.

47. Benaboud S, Pruvost A, Coffie PA, Ekouévi DK, Urien S, Arrivé E, et al. Concentrations of tenofovir and emtricitabine in breast milk of HIV-1-infected women in Abidjan, Côte d'Ivoire, in the ANRS 12109 TEmAA Study, step 2. Antimicrob Agents Chemother. 2011;55(3):1315-7.

48. Brown RS, Verna EC, Pereira MR, Tilson HH, Aguilar C, Leu CS, et al. Hepatitis B virus and human immunodeficiency virus drugs in pregnancy: Findings from the Antiretroviral Pregnancy Registry. J Hepatol. 2012;57(5):953-9.

49. Ayoub WS, Cohen E. Review Article Hepatitis B Management in the Pregnant Patient: An Update. J Clin Transl Hepatol. 2016;4(1):241-7.

50. Hu Y, Chen J, Wen J, Xu C, Zhang S, Xu B, et al. Effect of elective cesarean section on the risk of mother-to-child transmission of hepatitis B virus. BMC Pregnancy Childbirth. BMC Pregnancy and Childbirth; 2013;13(1):119.

51. Yang J, Zeng X, Men Y, Zhao L. Elective caesarean section versus vaginal delivery for preventing mother to child transmission of hepatitis B virus - a systematic review. Virol J. 2008;5:100.

52. Visvanathan K, Dusheiko G, Giles M, Wong ML, Phung N, Walker $\mathrm{S}$, et al. Managing HBV in pregnancy. Prevention, prophylaxis, treatment and follow-up: position paper produced by Australian, UK and New Zealand key opinion leaders. Gut. 2016;65(2):340-50. 